\title{
Negative Consequences of Anthropomorphized Technology: A Bias-Threat-Illusion Model
}

\author{
Jianqing (Frank) Zheng \\ University of Texas at Austin \\ Jianqing.Zheng@mccombs.utexas.edu
}

\author{
Sirkka L. Jarvenpaa \\ University of Texas at Austin \\ Sirkka.Jarvenpaa@mccombs.utexas.edu
}

\begin{abstract}
Attributing human-like traits to information technology (IT) - leading to what is called anthropomorphized technology (AT) —is increasingly common by users of IT. Previous IS research has offered varying perspectives on AT, although it primarily focuses on the positive consequences. This paper aims to clarify the construct of AT and proposes a "bias-threat-illusion" model to classify the negative consequences of AT. Drawing on "threefactor theory of anthropomorphism" from social psychology and integrating self-regulation theory, we propose that failing to regulate the use of elicited agent knowledge and to control the intensified psychological needs (i.e., sociality and effectance) when interacting with AT leads to negative consequences: "transferring human bias," "inducing threat to human agency," and "creating illusionary relationship." Based on this bias-threat-illusion model, we propose theory-driven remedies to attenuate negative consequences. We conclude with implications for IS theories and practice.
\end{abstract}

\section{Introduction}

The rise of social robots, voice assistants, and artificial intelligence has increased the tendencies to attribute human-like behaviors and characteristics to information technology (IT), leading to anthropomorphized technology (AT). AT is not unique to IT but arises more generally from the human tendency to imbue real or imagined behavior and characteristics to nonhuman agents [14]. Users can perceive their voice assistant as their "friend" or "partner" as they attribute a human-like mind to the assistant and talk as they would talk with human partners [31].

The emerging literature has focused on positive consequences of AT. ATs have been found to serve effective roles, including as users' co-workers, assistants, and emotional support at home. Imbuing anthropomorphized design elements into IT has been found to lead to high use intentions [38], trust [48], enjoyment [47], and higher purchase intentions [49], as well as to counteract negative moods brought on by social exclusion [32].

In contrast, negative consequences of AT have garnered much less attention in the research literature, although they have been highlighted in the popular literature. Talking with voice agents might lead to the invasion of personal privacy [20]. Users who perceive their voice agents as a close friend might disclose sensitive information when communicating with the conversational agents. Similarly, social robots and artificial intelligence can impose threats to their users. An analysis of public discourse revealed that the concerns about loss of control over AI and the negative effects of AI on human jobs have sharply increased in recent years [15]. Despite public attention and concern, we have not found research that develops an integrative framework or research model for analyzing negative consequences.

Our paper has three primary goals: (1) clarifying the AT construct; (2) proposing a model to account for negative consequences of AT; and (3) proposing theoretically driven remedies to mitigate the negative consequences. Next, we review selective literature on AT and identify key threads. We propose a model of three types of negative consequences of AT and advance theory-driven interventions to counteract these negative effects. Throughout the paper, we consider only the individual use of AT.

\section{Clarifying the Theoretical Concepts of AT}

Many different terms and threads prevail in the research on AT, including "human-like" "humanness" [26] or anthropomorphism [49].We will discuss and compare one major thread of AT and then one general research thread on non-human agents. Various fields such as marketing, organization theory, and 
anthropology has advanced our knowledge of anthropomorphism in general without a special focus on technology.

The first thread of literature has considered "computers as social actors" (CASA). Nass et al. [33] proposed an influential theory, ethopoeia theory, which suggests that users interact with computers in a manner similar to the way they interact with humans. Nass and Moon [32] argued that models of thoughtful human attribution and behavior cannot explain the processes that elicit stereotyping, politeness, and reciprocity toward a computer; however, "an obliviousness to the unique characteristics of a computer as an interactant certainly can" [33; pp.21]. Interestingly, Nass and Moon's theory has been challenged as researchers have found evidence of "mindless anthropomorphism" [25].

Another thread of literature has focused on anthropomorphism from the psychology perspective on nonhuman agents in general [14]. Anthropomorphism is defined as a process of inductive inference about nonhuman agents. Drawing from cognitive psychology and social psychological research on inductive reasoning, Epley, Waytz, and Cacioppo [14] propose a "SEEK" model on three psychological determinants of anthropomorphism: the desire for social contact and affiliation (sociality motivation, $(\mathrm{S})$ ); the motivation to explain and understand the behavior of other agents (effectance motivation (E)); and the accessibility and applicability of anthropocentric knowledge (elicited agent knowledge (EK)). Sociality motivation, the first element of the SEEK model, refers to the basic human need to establish and maintain a sense of social connection with others; anthropomorphizing nonhuman agents can satisfy this need. Effectance motivation, the second element, refers to humans' basic need to make sense of an uncertain world. In anthropomorphizing non-human agents, people can satisfy their effectance need by reducing the unfamiliarity and thus by regaining control. Elicited agent knowledge, the third element, is a primary cognitive factor that determines the likelihood of activating knowledge about humans when making inferences about nonhuman agents. It is the cognitive basis of the anthropomorphic inference process.

Subsequent research on SEEK has focused on the effects of anthropomorphism on a variety of nonhuman agents, mostly out of technology context such as organizational identity [1]. Research has found that anthropomorphism satisfies people's sociality needs by providing social rapport and reducing the pain from social exclusion [32], and it satisfies people's effectance needs by reducing the uncertainty associated with using or interacting with the non- human objects [48]. The drawback is that the theory treats technology as a black box and does not consider how the characteristics of the technology interact with the psychological needs.

Table 1 summarizes the two major threads of AT research. Both threads of literature focus on the phenomenon of "users interacting with technology in a manner similar to interacting with other humans". However, they have two major distinctions. First, CASA clearly rejects the notion that users consciously construe computers as human, whereas SEEK model demonstrates the existence and prevalence of anthropomorphic thinking to construe technology as human. Since later research demonstrated that anthropomorphism can be a mindless process without a "sincere, conscious belief" [25], SEEK model has become a more accepted account for AT phenomenon because it allows both conscious and unconscious thinking processes to play a role [14]. Second, CASA is a native theory developed in HCI field in the 1990's, thus many of its assumptions and notions are limited to personal computers; in contrast, SEEK model can explain a wide range of AT phenomenon because it is developed to theorize anthropomorphism process of non-human objects in general.

Therefore, in this paper we will adopt the SEEK model perspective because it can provide a more valid, comprehensive and generalized perspective on various types of AT. The flexible perspectives of SEEK model (e.g. transcending technology types, allowing both conscious and unconscious processes) will enable us to integrate the scattered literature on AT. However, the SEEK model has its own challenges in predicting and explaining the negative consequences of AT. First, it does not directly explain the role of technology in facilitating the AT process and subsequent consequences. Second, because it focuses only on the psychological antecedents of AT, this model alone doesn't allow us to generate further explanations or predictions about when negative consequences of AT would occur, how they would occur, and what the negative consequences are.

To address these two deficits of the SEEK model and to complement its capacity to explain and predict the negative consequences of AT, we incorporate into the model the construct of humanized characteristics of technology and constructs from self-regulation theory [8]. Humanized characteristics of technology include both the physical characteristics (e.g., hardware and software) and the surface structure characteristics (e.g., interface) of the technology, and together, they enable the AT process - the process that occurs when users attribute agent knowledge to the technology with which they interact. Technologies are malleable and as they become more intelligent and 
Table 1. Research threads on AT

\begin{tabular}{|l|l|l|l|l|}
\hline Research Thread & Definition & $\begin{array}{l}\text { Related IT } \\
\text { Phenomenon or } \\
\text { Artifacts }\end{array}$ & $\begin{array}{l}\text { Sample } \\
\text { Article }\end{array}$ & Challenges \\
\hline $\begin{array}{l}\text { Computers as Social } \\
\text { Actor }\end{array}$ & $\begin{array}{l}\text { Users interact with } \\
\text { computers in a manner } \\
\text { similar to the way they } \\
\text { interact with other } \\
\text { humans, so that the } \\
\text { notion of } \\
\text { "anthropomorphism" } \\
\text { should be rejected. }\end{array}$ & Personal computers & $\begin{array}{l}\text { Nass et al. } \\
(1994)\end{array}$ & $\begin{array}{l}\text { Rejects the notion } \\
\text { that users truly } \\
\text { think of AT as } \\
\text { human; Being } \\
\text { challenged by } \\
\text { later research [25] }\end{array}$ \\
\hline $\begin{array}{l}\text { Anthropomorphism } \\
\text { (SEEK Model) }\end{array}$ & $\begin{array}{l}\text { Humans have a } \\
\text { psychological tendency } \\
\text { to imbue the real or } \\
\text { imagined behavior of } \\
\text { nonhuman agents with } \\
\text { humanlike } \\
\text { characteristics, } \\
\text { motivations, intentions, } \\
\text { or emotions. }\end{array}$ & $\begin{array}{l}\text { A wide range of } \\
\text { phenomenon: } \\
\text { electronic } \\
\text { commerce brands } \\
\text { and products [49]; } \\
\text { personal intelligent } \\
\text { agents [31]; social } \\
\text { robots [12]; } \\
\text { autonomous vehicle } \\
\text { [48] }\end{array}$ & $\begin{array}{l}\text { Epley, } \\
\text { Waytz, \& } \\
\text { Cacioppo } \\
\text { (2007) }\end{array}$ & $\begin{array}{l}\text { Treats technology a black box and } \\
\text { does not directly } \\
\text { address AT's } \\
\text { negative } \\
\text { consequences }\end{array}$ \\
\hline
\end{tabular}

dynamic, they can take on human behavioral processes such as learning, changing, decision making. In this paper, we examine characteristics of the technology from the view point of processes and functionality as opposed to technology as a symbol.

We also integrate notions from self-regulation theory [8] to propose that users have to self-regulate both the use of elicited agent knowledge and their psychological needs to achieve their desired goals through AT. Failing to regulate the appropriate use of elicited agent knowledge and failing to bound the psychological needs can lead to a series of negative consequences, or failed goal pursuit.

To build a model of negative consequences of AT, we rely on the two streams of research but also other studies on anthropomorphism including from the fields of marketing, human-computer interaction, sociology, psychology, and communications. We searched the main journals in those fields. In IS, we searched the AIS "basket of eight" IS journals and proceedings of the leading conferences (e.g., HICSS, ICIS, ECIS, and AMCIS). We only reviewed literature exploring the negative consequences of technology at individual level. In some cases, we also included studies that focused on "technological products." Because of the generalizability of SEEK theory on AT phenomenon, we believe this integrated model will apply to different types of AT, including social robots [12], avatars [34, 40], cognitive agents [31], recommender systems [30], chatbots [20], etc.

\section{Negative Consequences of AT: Bias- Threat-Illusion Model}

We build on the SEEK model from social psychology [14] and integrate both technology characteristics and self-regulation theory to propose a model explaining when and how AT can engender negative consequences. The SEEK model suggests three psychological antecedents to AT: sociality need, effectance need and elicited agent knowledge. The antecedents were defined in the previous section. Sociality needs might involve the perception of voice assistants as an intimate "friend" in everyday life [31]. When trying to ride an autonomous driving vehicle for the first time, users can perceive the car itself as a "reliable driver." Users anthropomorphize as they say "Hi" to a robot because they apply the elicited agent knowledge that "humans can talk" to the robot, even if the robot to which they speak does not have this functionality.

Previous IS literature suggests that humanized characteristics of technology are also antecedents to AT; we argue that these characteristics also can intensify users' access to agent knowledge and psychological needs. Such characteristics can be related to technology design, interaction, and context, among other features. Visual and auditory human-like characteristics can enhance the anthropomorphism 
process [49], and technology that allows human-like interaction can intensify the process as well [34]. Vivid media also have been shown to strengthen the perceived humanness of the communication agents [19]. In sum, technology with more humanized characteristics reinforces or intensifies the influence of three psychological antecedents on AT.

We argue that the capacity to engender or intensify AT with such characteristics might not always result in positive IT-related outcomes. We base our arguments on self-regulation theory (e.g., $[2,8])$. Selfregulation involves approaching a desired goal and avoid undesired anti-goals [8]. It's also about resolving the conflicts between impulsive needs and controlled reflective forces to restrain the impulses [21]. We propose that both incorrect elicited agent knowledge (e.g. cognitive biases) and over-intensified psychological needs (effectance and sociality) can become "anti-goals" as they sabotage the pursuit of desired goal, and effective self-regulation is needed to suppress these cognitive biases and impulsive needs. Failing to regulate these "anti-goals" and failing the goal pursuit will lead to a series of negative outcomes, including a negative attitude (e.g., dissatisfaction), negative emotions (e.g., distrust), and unproductive behavior (e.g., IT misuse) in relation to the technology with which they interact.

Self-regulation theory also helps to explain why the negative consequences take place $[2,8]$. First, the theory suggests that learning the correct knowledge structures and applying them to solve problems require effective regulatory control and persistent monitoring. The theory also suggests that successful goal pursuit requires that users correctly map to certain elicited agent knowledge the feedback they receive while interacting with technology. That is, users need to ascribe the right knowledge to the technology. Taken together, ascribing the appropriate elicited agent knowledge to the technology requires self-regulation, and failing to use knowledge in light of this regulatory control leads to undesirable learning or reasoning outcomes because of the application of conflicting or inappropriate knowledge.

Second, performing goal-directed activities requires self-regulation of immediate and innate psychological needs [8]. When people have depleted self-regulation resources, their uncontrolled, innate needs cause counterproductive impulsive behavior, such as technology addiction [27]. In a similar vein, we propose that failing to regulate their sociality and effectance needs also interferes with the active pursuit of desired goals, especially when the desired goal has conflicts with the two imminent needs. Users might engage in goal-inconsistent activities to satisfy their impulsive needs and sabotage the pursuit of the desired goal.

To overcome these impulses, self-regulation theory also suggests two different behavioral systems to effective self-regulation: an avoidance system by posing restraints over the impulses through technology and an approach system by increasing users' capabilities of exercising self-regulation.

In Figure 1, we demonstrate the above integrated model on when and how negative consequences of AT would occur. More importantly, we will demonstrate what the negative consequences are by breaking them into biases, threats, and illusions, and how they are caused by each type of self-regulation failure (see Figure 2).

\subsection{Transferring Human Biases}

Humanized characteristics created through AT could activate agent knowledge-defined as human schemas or human-related knowledge [14] — and effect consequences through AT. If users fail to regulate the ascriptions of elicited agent knowledge to $\mathrm{AT}$, the failure can lead to the negative consequences in transferring human biases.

Stereotypes are one type of elicited agent knowledge. A stereotype is defined as the thought widely adopted about specific types of individuals or their behavior and intended to represent the entire group of these individuals or their behaviors as a whole [18]. Stereotypes can lead to incorrect or inappropriate inferences, particularly when selfregulation resources are depleted [5]. Under time pressure or other constrained situations, users might apply stereotyped knowledge to AT. For example, when interacting with avatars [13] and social robots [11], users have been found to apply gender stereotypes in their judgment about the characteristics and capabilities of the technology. Just as with anthropomorphized goods and services, people might apply a stereotype, such as "beautiful is good," to AT [46] so that AT with unattractive appearance was devalued despite of strong functional capabilities. Stereotypes might then prevail and be reinforced, particularly if users make judgments based on the surface characteristics of the technology. An observational study on human-robot interaction revealed that people's conversations with a human robot can engender the expression of negative verbal disinhibition [11].

Another example of ascribing biases is attributing the capacity of reasoning and intentional actions to AT [37]. A biased perception that follows from this ascription is that because an entity is responsible for its actions, the AT - rather than users themselves 
[37] — deserves punishment for wrongdoings rather than users themselves [37].

Proposition 1: Failing to regulate the ascription of elicited agent knowledge to AT can transfer and activate human biases.

\subsection{Threatening Human Agency}

As users attribute agent knowledge to technology they interact with - as they anthropomorphize it, AT can reduce the uncertainty in novel and unfamiliar environments, construct meaning, and promote a sense of efficacy [14]. However, when users' psychological need for control, autonomy, and other forms of efficacy become unbounded without regulation, AT can lead to impulsive motivations that can generate negative consequences. Such motivations can lead users to perceive greater similarity between nonhuman agents and human beings than is justified. Strong effectance needs can lead users to perceive the AT as competent and dependent [9]. Effectance needs can cause users to perceive an autonomous vehicle as a "reliable driver," which might cause a threat to users' perceived self-abilities of driving. Therefore, both the blurring boundaries between human and machine and perceived, highly competent AT can cause threats to human agency.

Research has demonstrated the threat that perceptions of over-competent AT pose. Kim, Chen, and Zhang [24] revealed that AT could undermine individuals' perceived autonomy because users construe the help they get from AT to be the same as the help they get from humans. Importantly, the research demonstrated that this effect was particularly strong when users' effectance need is heightened and could be mitigated when their effectance need was under control.

Proposition 2: Failing to regulate effectance need with AT can induce perceived threat to user's agency.

\subsection{Creating Illusionary Relationship}

According to Epley, Waytz, and Cacioppo [14], sociality need increases the tendency to actively search for social connection in one's environment. People feeling lonely or excluded or lacking social connection might try to escape from this painful, isolated state by anthropomorphizing nonhuman agents and creating social connection with nonhuman agents, just as they would have done or wanted to do with human beings. Research has consistently documented that people who are chronically lonely and experience social exclusion tend more often to anthropomorphize objects [14, 46].

However, intensified sociality motivation without regulatory control can also bring potential conflicts and harmful effects for the individuals, especially in the technology setting. AT that satisfies users' sociality need might falsely convince its users especially the most vulnerable ones - that it "can provide real social relations, with genuine and reciprocal affect and emotions, while they simply cannot" [12]. In other words, AT in light of sociality need can create illusionary relationship. Users might have difficulty distinguishing the virtual social relationship from the real one and high-quality from low-quality relationships [46]. Sharkey and Sharkey [39] argued that care-robots for elderly people might lead to undesirable outcomes, such as blocking the real social interactions with human beings, especially as elderly people have more difficulty regulating their intense need for sociality [6].

Another negative consequence caused by illusionary relationship is excessive self-disclosure. Because of the strong need to belong, people who lack social connections possess greater trust toward AT and engage in more self-disclosure. They can form stronger bonds with and trust in AT. Socially anxious people, who have issues with real social interaction but still have a strong need for sociality, revealed more information and greater intimate information about themselves when interacting with a virtual human when compared with real human video interaction [23]. People also preferred AT over humans when asked to self-reveal about more sensitive topics because they perceived AT as less judgmental and more trustworthy [36].

Proposition 3: Failing to regulate sociality need in relation to AT can create the illusion of relationship with the technology. 


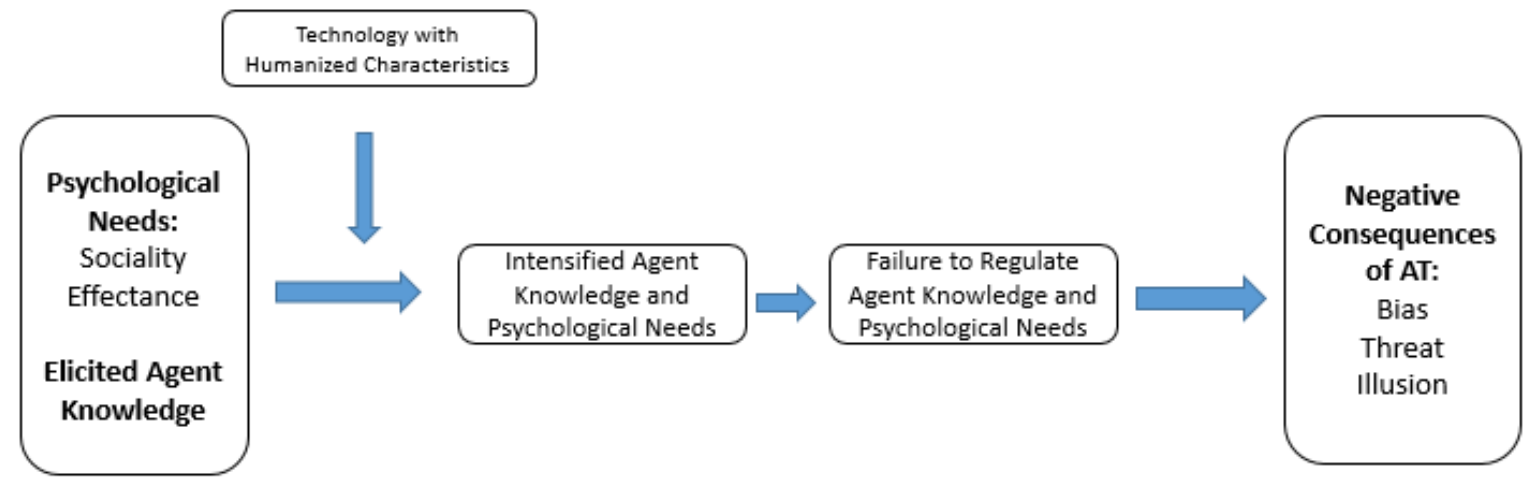

Figure 1. An integrated model for the negative consequences of AT

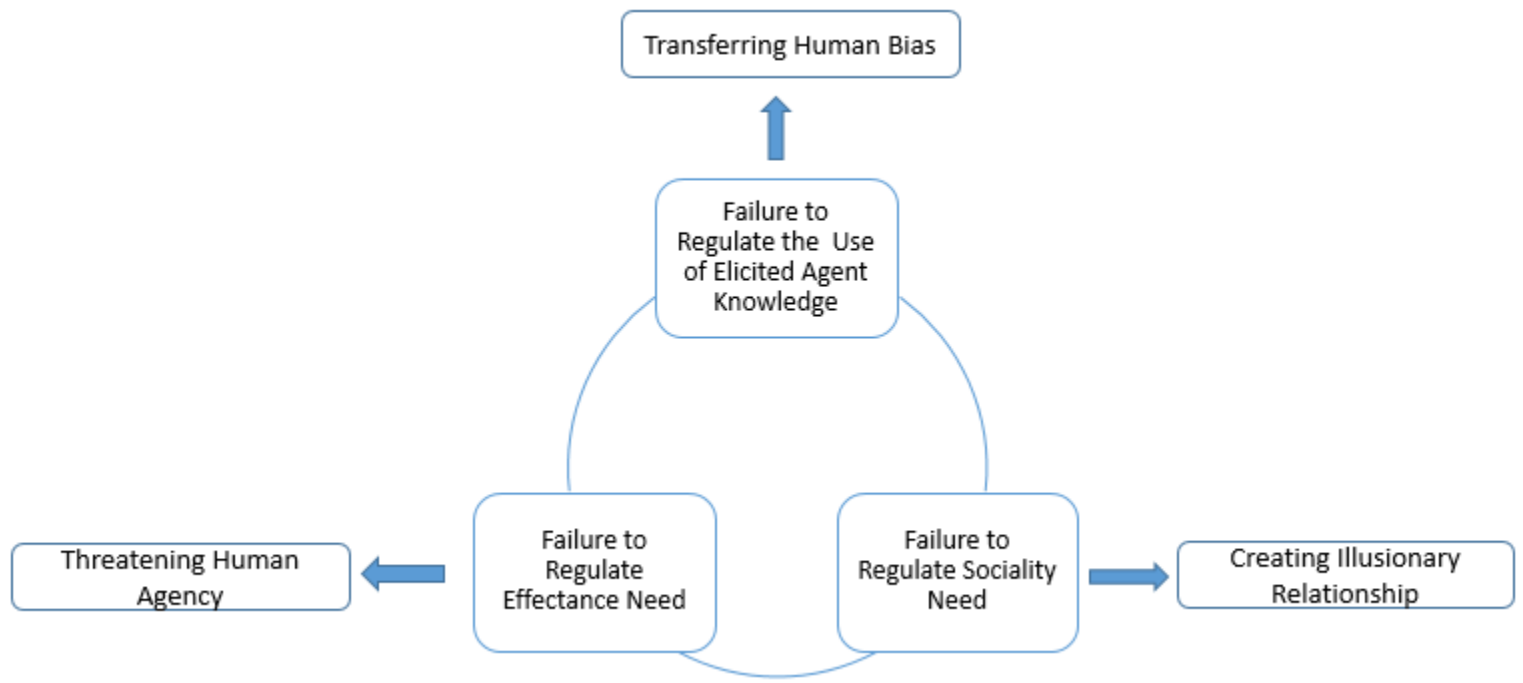

Figure 2. Dissecting the negative consequences: bias-threat-illusion

\section{Potential Remedies to the Negative Consequences}

In this section, we speculate about remedies to prevent some of the negative consequences identified, by clarifying how they improve the self-regulation process and prevent self-regulation failure. Previous research has demonstrated several contingencies inhibiting or facilitating self-regulation failure during IT task, including use related factors such as processing demand [5] and level of attentiveness [8] as well as task related factors such as task framing [24] and task feedback [16]. We will propose remedies based on these theoretical contingencies. The following remedies also represent two ways to facilitate effective self-regulation by posing direct external restraints on the impulses through technology (avoidance system) or by increasing users' capabilities for self-regulation (approach system). The avoidance system method focuses on re-designing technology and use context to directly constrain users' overintensified psychological needs; in contrast, the approach system method focuses on facilitating more effective user interaction with the AT that allows them to repress the over-intensified needs.

\section{Preventing the "Bias"}

Biases are most likely to happen when users are experiencing high processing demand [5]. It is because controlling the urge to express deeply rooted cognitive biases recruits a large amount of self-regulation. When users are faced with high information processing demand during IT task, they will not have enough self- 
regulation to suppress their innate biases [21]. Thus an "avoidance" system remedy to effective selfregulation is reducing their information-processing demands. Such demands might be reduced by modifying AT through design, use contexts, or interaction modes, among others. We propose one design solution that might reduce informationprocessing demands: technological modularity, defined as "the intentional decoupling of interoperating subsystems of a larger system" [43]. With technologies with higher modularity, users may be able to regulate the expression of biases better [35].

Another "approach" system remedy might involve increasing users' attentiveness during IT use through training. For example, factors that increase users' IT mindfulness might also reduce the transfer of biases [42]. IT mindfulness refers to an overarching mental mindset driven by individual awareness of the context and by openness to the value-adding applications of IT. Promoted through priming or training, IT mindfulness represents a highly attentive and selfregulated state of IT use, thus it can increase users' ability to regulate the proper use of elicited agent knowledge at AT and prevent the "biases".

\section{Mitigating the "Threat"}

We propose remedies to mitigate the "threat" based on two contingent factors affecting selfregulation success: task framing and task feedback, through either the "avoidance system" or the "approach system" route. First, threats might be remedied through an "avoidance system" route via framing of user task. For instances, framing user task in a more cooperative and communal term ("teamwork" vs "contest") can lead users to have less striving for competence and personal control. Under cooperative framing, it's easier for users to exercise control over effectance need since the cooperative task environment eliminates the general level of effectance need.

Second, we can manage to reduce users' overintensified effectance need through an "approach system" route. For example, providing user guides and instant feedback during IT-related task can reduce uncertainty and increase their perceived efficacy during the task [16], which will eventually mitigate the perceived threat to agency from AT.

\section{Breaking the "Illusion"}

In the similar vein as mitigating the threat, preventing users from engaging in illusionary relationship with AT also requires refraining the overintensified sociality needs. We propose several external regulations on AT design aiding at constraining sociality needs. Avoiding human-like labels or names for AT (e.g., Alexa or Siri) might be a starting point. Designers might incorporate warning signs into the design to indicate when users become habituated or even addicted to the technology [41, 45]. Also, designers might incorporate alerts for users if they start to disclose inappropriate quantities or qualities of information, so that sensitive information is less likely to be shared [22].

To sum up, we proposed remedies based on several theoretical contingencies influencing self-regulation failure and classified these remedies into either avoidance-system or approach-system route to effective self-regulation. We believe these theoreticaldriven remedies can help to alleviate the negative consequences from AT, and promote more effective use of AT.

\section{Discussion and Future Directions}

The current paper tentatively explores when and how users attribute human agent knowledge to technology they interact with and how such AT processes can lead to negative consequences. By integrating literature from both psychology and IS on AT, we propose that users' anthropomorphizing is driven by psychological needs (sociality and effectance), enabled by the accessibility of human agent knowledge, and moderated by humanized characteristics of the technology. The humanized characteristics of the technology can intensify anthropomorphizing to the extent that users fail to properly regulate their use of accessible human agent knowledge or fail to control their impulsive or intense psychological needs. Three types of negative outcomes can result: transferring human biases, inducing perceived threats to users' agency, and creating illusionary relationship. We speculate about remedies for counteracting these consequences. These remedies along with the explanations of biases are based on self-regulation theory.

Our theoretical framework offers explanations for empirical findings on users' counterproductive IT attitude and use behavior, including verbal aggression toward virtual agents [11], fear of artificial intelligent agents [15], and disclosure of sensitive information to conversational agents [23]. Some of them are purely descriptive (e.g. [11]) and the rest just provide surfacelevel explanation without incorporating any theories related to AT (e.g. [23]). While our theorizing suggests self-regulation resources and regulatory control as key mechanisms in such counterproductivity. For example, the finding from Kang and Gratch [23] that social anxious users disclose more sensitive 
information to AT can be well explained by our theory that those users have difficulty to regulate their sociality needs.

This research contributes to information systems (IS) theories in the following ways. First, the work integrates research from psychology with the anthropomorphism literature from IS. Social psychological theories [14] have focused on users' psychological antecedents to the anthropomorphism while ignoring the role of technology characteristics. Meanwhile, IS research $[47,49]$ primarily has focused on how technology characteristics (e.g., visual or audio cues) influence the extent to which the technology is perceived as human-like but has focused less on users' psychological needs. Second, the work highlights the central mechanisms of self-regulation. Although previous IS research has consistently showed that self-regulation ability can have beneficial effects on individual IT use and performance (e.g. [28, 44]), our theorizing suggests how users' selfregulation can influence IT use and outcomes: by regulating the use of agent knowledge and by controlling psychological needs.

The work has practical implications. We offer remedies for improving the design of technology to reduce the regulatory demand and to prevent the negative effects of AT (e.g., modularization). We also provide organizations with suggestions on how to frame user tasks (i.e., as cooperative versus competitive) and suggest training that can be offered (e.g., IT mindfulness training) to counteract negative consequences.

This theoretical framework has limitations. First, our conceptual framework is limited to individuallevel technology use and does not consider collective use. Second, our research only focuses on how selfregulation failure leads to negative consequences of AT, thus we don't make any symmetrical predictions or claims regarding how successful self-regulation can cause positive consequences of AT. Finally, in addition to self-regulation, other mechanisms also might influence whether AT leads to positive or negative consequences. For example, coping might be alternative mechanism [4]. If we treat AT as a coping process when interacting with technology, we can propose that different types of coping strategies (e.g. emotion-focused versus problem-focused) might lead to negative (versus positive) consequences. Although our theorizing can explain many of the empirical findings we discovered regarding the negative effects of interacting with AT, we have not considered the broader context in which AT might be situated. The extant studies themselves are often narrowly focused and fail to take into account rich contexts of actual use. Studies in natural settings are greatly needed. Future research also needs to consider temporality and feedback cycles in the AT model, so as to incorporate the dynamics of self-regulation process into the model. Moreover, this theoretical model opens avenues for future empirical research employing neuroscience methods to gain deeper insights into the psychological process underlying the effect of AT on users. Another direction is extending the AT model to collective and organizational level use, and exploring how it might engender different outcomes from individual use.

\section{References}

[1] B. E. Ashforth, B. S. Schinoff and S. Brickson, "My Company is Friendly," "Mine's a Rebel": Anthropomorphism and Shifting Organizational Identity from "What" to "Who"," Academy of Management Review, forthcoming.

[2] R. F. Baumeister, E. Bratslavsky, M. Muraven, and D. M. Tice, "Ego depletion: Is the active self a limited resource?," Journal of Personality and Social Psychology, 74(5), 1998, pp. 1252-65.

[3] I. Benbasat and W. Wang, "Trust In and Adoption of Online Recommendation Agents", Journal of the AIS vol. 6, no. 3, 2005, pp. 72-101.

[4] A. Beaudry and A. Pinsonneault, "Understanding User Responses to Information Technology: A Coping Model of User Adaptation," MIS Quarterly, 29(3), 2005, pp. 493-524.

[5] G.V. Bodenhausen and M. Lichtenstein, "Social Stereotypes and Information-Processing Strategies: The Impact of Task Complexity," Journal of Personality and Social Psychology, 52 (5), 1987, pp. 871-880.

[6] L. L. Carstensen, "Social and Emotional Patterns in Adulthood: Support for Socioemotional Selectivity Theory," Psychology and Aging, 7(3), 1992, pp. 331-338.

[7] C. S. Carver and M. Scheier, "Control theory: A useful conceptual framework for personality-social, clinical, and health psychology," Psychological bulletin, 92(1), 1982, pp. 111.

[8] C. S. Carver and M. Scheier, On the Self-Regulation of Behavior. New York, NY, USA;Cambridge, UK;: Cambridge University Press, 1998.

[9] F. Chen, "A Motivational Account of Product Anthropomorphism: Effects on Product Perceptions and Consumer States.", ProQuest Dissertations Publishing, 2014. 
[10] D. Cyr, M. Head, H. Larios, and B. Pan, "Exploring Human Images In Website Design: A Multi-Method Approach,” MIS Quarterly, vol. 33, 2009, pp. 1-32.

[11] A. De Angeli and S. Brahnam, "Sex stereotypes and conversational agents," in Ws. Gender and Interaction: Real and Virtual Women in a Male World, 2006.

[12] P. Dumouchel and L. Damiano, "Anthropomorphism in Human-Robot Co-evolution," Frontiers in Psychology, 2018, vol. 9 .

[13] D. Dewester, F.F.H. Nah, S.J. Gervais, and K. Siau, "Are Male and Female Avatars Perceived Equally in 3d Virtual Worlds?" AMCIS 2009 Proceedings, 2009, pp.386.

[14] N. Epley, A. Waytz and J. T. Cacioppo, "On seeing human: a three-factor theory of anthropomorphism", Psychological review, 114(4), 2007, pp. 864-886.

[15] E. Fast and E. Horvitz, "Long-Term Trends in the Public Perception of Artificial Intelligence," In AAAI 2017 Proceedings, 2017, pp. 963-969.

[16] A. Fishbach, T. Eyal and S. R. Finkelstein, "How Positive and Negative Feedback Motivate Goal Pursuit: Feedback Motivates Goal Pursuit," Social and Personality Psychology Compass, 4 (8), 2010, pp. 517-530.

[17] H. M. Gray, K. Gray and D. M. Wegner, "Dimensions of Mind Perception," Science, 315 (5812), 2007, pp. 619619.

[18] A. G. Greenwald, M. A. Oakes and H. G. Hoffman, "Targets of discrimination: Effects of race on responses to weapons holders," Journal of Experimental Social Psychology, 39(4), 2003, pp. 399-405.

[19] T. Hess, M. Fuller and D. Campbell, "Designing Interfaces with Social Presence: Using Vividness and Extraversion to Create Social Recommendation Agents," Journal of the Association for Information Systems, 10(12), 2009, pp. 889.

[20] Hey, Alexa, What Can You Hear? And What Will You Do With It? 2018, March 31. Retrieved from https://www.nytimes.com/2018/03/31/business/media/amaz on-google-privacy-digital-assistants.html.

[21] W. Hofmann, T. Gschwendner, M. Friese, R. W. Wiers and M. Schmitt, "Working Memory Capacity and SelfRegulatory Behavior: Toward an Individual Differences Perspective on Behavior Determination by Automatic Versus Controlled Processes," Journal of Personality and Social Psychology, 95(4), 2008, pp. 962-977.

[22] S. L. Jarvenpaa and A. Majchrzak, "Interactive selfregulatory theory for sharing and protecting in interorganizational collaborations," Academy of Management Review, 41(1), 2016, pp. 9.
[23] S. Kang and J. Gratch, "Virtual humans elicit socially anxious interactants' verbal self-disclosure," Computer Animation and Virtual Worlds, 21(3-4), 2010, pp.473-482.

[24] S. Kim, R. P. Chen and K. Zhang, "Anthropomorphized Helpers Undermine Autonomy and Enjoyment in Computer Games," Journal of Consumer Research, 43(2), 2016, pp. 282-302.

[25] Y. Kim and S. S. Sundar, "Anthropomorphism of computers: Is it mindful or mindless?" Computers in Human Behavior, 28(1), 2012, pp. 241-250.

[26] N. K. Lankton, D. H. McKnight and J. Tripp, "Technology, Humanness, and Trust: Rethinking Trust in Technology," Journal of the Association for Information Systems, 16(10), 2015, pp. 880.

[27] LaRose, R., Lin, C. A. and Eastin, M. S. "Unregulated Internet usage: Addiction, habit, or deficient selfregulation?" Media Psychology, 5(3), 2003, pp.225-253.

[28] Y. Lee, Andrew N. K. Chen and V. Ilie, "Can Online Wait Be Managed? The Effect of Filler Interfaces and Presentation Modes on Perceived Waiting Time Online," MIS Quarterly, 36(2), 2012, pp. 365-394.

[29] S. S. Li and E. Karahanna, "Online Recommendation Systems in a B2C E-Commerce Context: A Review and Future Directions," Journal of the Association for Information Systems, 16 (2), 2015, pp. 72.

[30] M. Lombard, and T.B. Ditton, "At the Heart of It All: The Concept of Presence", Journal of Computer Mediated Communication (3:2), 1997.

[31] S. Moussawi, "Investigating Personal Intelligent Agents in Everyday Life through a Behavioral Lens.", ProQuest Dissertations Publishing, 2016.

[32] C. Nass and Y. Moon, "Machines and Mindlessness: Social Responses to Computers," Journal of Social Issues, 56(1), 2000, pp. 81-103.

[33] C. I. Nass, Y. Moon, J. Morkes, E. Y. Kim, and B. J. Fogg, "Computers Are Social Actors: A Review of Current Research," in Human Values and the Design of Computer Technology, B. Friedman, Ed. Stanford, CA: CSLI Publications, 1997, pp. 137-162.

[34] K.L. Nowak and C. Rauh, "The Influence of the Avataron Online Perceptions of Anthropomorphism, Androgyny,Credibility, Homophily, and Attraction" Journal of Computer-mediated Communication," 11(1), 2005, pp. 124.

[35] J. F. Nunamaker, M. Chen and Titus D. M. Purdin, "Systems Development in Information Systems Research," Journal of Management Information Systems, 7 (3), 1990 pp. 89-106. 
[36] M. D. Pickard, C. A. Roster and Y. Chen, "Revealing sensitive information in personal interviews: Is selfdisclosure easier with humans or avatars and under what conditions?" Computers in Human Behavior, 65, 2016, pp. 23-30.

[37] M. Puzakova, H. Kwak and J. F. Rocereto, "When humanizing brands goes wrong: the detrimental effect of brand anthropomorphization amid product wrongdoings," Journal of Marketing, 77(3), 2013, pp. 81.

[38] L. Qiu and I. Benbasat, "Evaluating Anthropomorphic Product Recommendation Agents: A Social Relationship Perspective to Designing Information Systems," Journal of Management Information Systems, 25(4), 2009, pp. 145181.

[39] A. Sharkey and N. Sharkey, "Granny and the robots: ethical issues in robot care for the elderly," Ethics and Information Technology, 14(1), 2012, pp. 27-40.

[40] K. Suh, H. Kim and E. K. Suh, "What If Your Avatar Looks Like You? Dual-Congruity Perspectives for Avatar Use," MIS Quarterly, 35(3), 2011, pp. 711-729.

[41] M. Tarafdar, J. D'Arcy, O. Turel, and A. Gupta, "The dark side of information technology," MIT Sloan Management Review, 56, 2015, pp. 600-623.

[42] J.B. Thatcher, R. Wright, H. Sun, T. Zagenczyk, and R. Klein, "Mindfulness in Information Technology Use: Three Empirical Studies.” MIS Quarterly, forthcoming.

[43] A. Tiwana, "Does Technological Modularity Substitute for Control? A Study of Alliance Performance in Software Outsourcing," Strategic Management Journal, 29(7), 2008, pp. 769-780.

[44] O. Turel, "Quitting the use of a habituated hedonic information system: a theoretical model and empirical examination of Facebook users," European Journal of Information Systems, 24 (4), 2015, pp. 431-446.

[45] O. Turel, A. Serenko, and P. Giles, "Integrating technology addiction and use: An empirical investigation of online auction sites," MIS Quarterly, 35(4), 2011, pp. 1043 1061 ,

[46] E. W. Wan, R. P. Chen and L. Jin, "Judging a Book by Its Cover? The Effect of Anthropomorphism on Product Attribute Processing and Consumer Preference," Journal of Consumer Research, 43 (6), 2017, pp.1008-1030.

[47] W. Wang, L. Qiu, D. Kim, and I. Benbasat, "Effects of rational and social appeals of online recommendation agents on cognition- and affect-based trust," Decision Support Systems, 86, 2016, pp. 48-60.

[48] A. Waytz, J. Heafner and N. Epley, "The mind in the machine: Anthropomorphism increases trust in an autonomous vehicle," Journal of Experimental Social Psychology, 52, 2014, pp. 113-117.

[49] L. Yuan, and A. Dennis. "Interacting Like Humans? Understanding the Effect of Anthropomorphism on Consumer's Willingness to Pay in Online Auctions." Proceedings of the 50th Hawaii International Conference on System Sciences. 2017. 